TRANSACTIONS OF THE

AMERICAN MATHEMATICAL SOCIETY

Volume 353, Number 8 , Pages 3371-3389

S 0002-9947(01)02786-6

Article electronically published on April 9, 2001

\title{
PROJECTIVE METRICS AND MIXING PROPERTIES ON TOWERS
}

\author{
VÉRONIQUE MAUME-DESCHAMPS
}

\begin{abstract}
We study the decay of correlations for towers. Using Birkhoff's projective metrics, we obtain a rate of mixing of the form:

$$
c_{n}(f, g) \leq \mathrm{Ct} \alpha(n)\|f\|\|g\|_{1}
$$

where $\alpha(n)$ goes to zero in a way related to the asymptotic mass of upper floors, $\|f\|$ is some Lipschitz norm and $\|g\|_{1}$ is some $L^{1}$ norm. The fact that the dependence on $g$ is given by an $L^{1}$ norm is useful to study asymptotic laws of successive entrance times.
\end{abstract}

\section{INTRODUCTION}

A powerful method to study nonuniformly hyperbolic systems or hyperbolic systems with singularities consists in conjugating the system to a tower (see the description of towers below). Indeed, this strategy was initiated by F. Hofbauer $([\mathrm{H}])$ to study piecewise expanding maps of the interval. It was then used to study piecewise expanding maps in higher dimension $(\mathrm{Buz})$, stochastic stability of unimodal maps $(([\mathrm{Ba}, \mathrm{V}],[\mathrm{Ba}, \mathrm{Y}])$, to obtain exponential decay of correlations for unimodal maps $([\mathrm{K}, \mathrm{N}, \mathrm{Y} 1])$, some billiards ([Y1], $[\mathrm{Ch}])$, Henon maps $([\mathrm{B}, \mathrm{Y}])$, partially hyperbolic diffeomorphisms ([D], Ca] $)$. In a recent paper, L.-S. Young ([Y2]) relates the decay of correlations for some mixing towers to the asymptotics of return times on the base of the tower. This gives, for example, polynomial decay of correlations for some interval maps with neutral fixed points. To this aim, she uses a coupling method.

We present a new approach to studying mixing properties on towers "à la Young". Using Birkhoff cones and projective metrics instead of coupling, we relate explicitly the rate of mixing to the mass of upper floors of the tower (Theorem 1.5). Moreover, our method gives strong mixing properties: the correlations depend of one observable solely through some $L^{1}$ norm. This is very useful to study asymptotic laws of return times and successive return times (see $[\mathrm{C}], \mathrm{C}, \mathrm{G}, \mathrm{S}, \mathrm{G}, \mathrm{S}],[\mathrm{Sau}, \mathrm{P}]$ ).

Section 1 contains the setting and precise statement of the results on decay of correlations (Theorem 1.5).

In section 2 we briefly recall definitions and properties of Birkhoff's cones and projective metrics.

Section 3 is devoted to the proof of the estimation of decay of correlations. The strategy is the following: we construct a sequence of cones of locally Lipschitz functions $C_{j}$ such that for some $k \in \mathbb{N}$ the transfer operator $\mathrm{E}^{k}$ maps $C_{j}$ into $C_{j+1}$

Received by the editors May 23, 1999 and, in revised form, January 13, 2000.

2000 Mathematics Subject Classification. Primary 37A25, 37C30, 37C40.

Key words and phrases. Decay of correlations, tower, transfer operator, projective metrics. 
with contraction $\gamma_{j}<1$. The rate of mixing is given by the product of the $\gamma_{j}$ 's. This product is easily related to the mass of the upper floors and may be subexponential. The rate of convergence of $\mathrm{E}^{k}$ to the spectral projection associated with the invariant density is obtained in the uniform norm on each floor.

In section 4 we discuss specific rates of mixing and lower bounds for uniform speed of convergence.

Acknowledgments. This article answers a question that Viviane Baladi posed to me. I thank her for this question and for many fruitful discussions on related topics. I also acknowledge support from the PRODYN program of the European Science Foundation.

\section{Setting, statement of the Results}

Let us describe the tower model. We follow Young's setting.

A tower $\Delta$ consists of floors $\Lambda_{\ell}, \ell \in \mathbb{N}$. The base $\Lambda_{0}$ of the tower is a probability space; let $m_{0}$ be the probability measure on $\Lambda_{0}$. The tower structure is given by a partition $\left(\Lambda_{0}^{j}\right)_{j \in \mathbb{N}}\left(\bmod m_{0}\right)$ of $\Lambda_{0}$ and a return time function defined on the base $\Lambda_{0}$ of the tower $R: \Lambda_{0} \longrightarrow \mathbb{N}$ which is constant on each $\Lambda_{0}^{j}$. The floors $\Lambda_{\ell}$ are just copies of a part of $\Lambda_{0}$ :

$$
\Lambda_{\ell}=\left\{(x, \ell) / x \in \Lambda_{0}, R(x)>\ell\right\} .
$$

We will denote by $\Lambda_{\ell}^{j}$ the copies of $\Lambda_{0}^{j}$ :

$$
\Lambda_{\ell}^{j}=\left\{(x, \ell) / x \in \Lambda_{0}^{j}, R(x)>\ell\right\} \subset \Lambda_{\ell} .
$$

The dynamic on the tower is given by $f_{0}: \Lambda_{0} \longrightarrow \Lambda_{0}$ such that $f_{0}: \Lambda_{0}^{j} \longrightarrow \Lambda_{0}$ is bijective $\left(\bmod m_{0}\right)$.

Remark 1.1. The assumption that $f_{0}: \Lambda_{0}^{j} \longrightarrow \Lambda_{0}$ is bijective may be replaced by the Markov assumption: $f_{0}\left(\Lambda_{0}^{j}\right)$ is a union of some $\Lambda_{0}^{k}$ 's and the large image property: $\exists \eta>0$ such that $\inf _{j \in \mathbb{N}} m_{0}\left(f_{0}\left(\Lambda_{0}^{j}\right)\right) \geq \eta$.

Let us consider $F: \Delta \longrightarrow \Delta$ defined by

$$
\left\{\begin{aligned}
F(x, \ell) & =F(x, \ell+1) \text { for }(x, \ell) \in \Lambda_{\ell} \text { and } R(x)>\ell+1 \\
& =f_{0}(x, 0)=: F^{R}(x, 0) \text { otherwise. }
\end{aligned}\right.
$$

On each floor $\Lambda_{\ell}$ we put the $\sigma$-algebra of $\Lambda_{0}$ pushed by $F$, so that $\Delta$ is a measurable space. We assume that the partition $\mathcal{R}=\left\{\Lambda_{\ell}^{j}\right\}$ generates in the sense that $\bigvee_{i=0}^{\infty} F^{-i} \mathcal{R}:=\left\{\bigvee_{i=0}^{\infty} F^{-i} A_{i}, A_{i} \in \mathcal{R}\right\}$, is the partition into points. For $k \in \mathbb{N}$, the elements of the partition $\mathcal{R}^{(k)}=\bigvee_{i=0}^{k-1} F^{-i} \mathcal{R}$ are called cylinder or $k$-cylinders. We denote by $C_{k}(x)$ the element of $\mathcal{R}^{(k)}$ which contains $x$.

The measure $m_{0}$ is pushed on each floor by $F$, we assume that $\int R d m_{0}<\infty$, so that we obtain a finite measure on $\Delta$. This measure will also be denoted by $m_{0}$ and we assume that it has been normalized $\left(m_{0}(\Delta)=1\right)$.

The space $\Delta$ may be endowed with a dynamical distance. For $x$ and $y$ in $\Lambda_{0}$, the separation time $s(x, y)$ is the greatest integer $n$ such that $\left(F^{R}\right)^{p}(x)$ and $\left(F^{R}\right)^{p}(y)$ belong to the same $\Lambda_{0}^{j}$ for all $p \leq n$. If $x$ and $y$ belong to $\Lambda_{\ell}, \ell \geq 1$, then they 
have exactly one preimage $x^{0}$ and $y^{0}$ in $\Lambda_{0}$, the separation time between $x$ and $y$ is $s(x, y)=s\left(x^{0}, y^{0}\right)$, if $x$ and $y$ are not in the same $\Lambda_{\ell}$, then $s(x, y)=0$. The distance on the tower is defined by : $d(x, y)=\beta^{s(x, y)}$ for some $0<\beta<1$. Endowed with this metric, the space $\Delta$ is separable (the topology is generated by the cylinders).

We will always assume the following regularity condition on $F$ :

$$
F^{R}: \Lambda_{0}^{j} \longrightarrow \Lambda_{0}
$$

and its inverse are nonsingular. Its Jacobian $J F^{R}$ satisfies the following bounded distortion inequality: $\exists C>0$ such that $\forall j \in \mathbb{N} \forall x, y \in \Lambda_{0}^{j}$

$$
\left|\frac{J F^{R}(x)}{J F^{R}(y)}-1\right| \leq C d\left(F^{R}(x), F^{R}(y)\right) .
$$

The Ruelle-Perron-Frobenius operator or transfer operator associated to $F$ is defined in the standard way:

$$
\mathrm{E}_{0} f(x)=\sum_{F(y)=x} J F(y)^{-1} f(y),
$$

where $J F$ is the Jacobian of $F$. Remark that except on $\Lambda_{0}$, this Jacobian is 1 . The bounded distortion property implies that for any $x \in \Lambda_{\ell}^{j}$ with $R(j)=\ell+1$,

$$
C^{-1} m_{0}\left(\Lambda_{\ell}^{j}\right) \leq J F(x)^{-1} \leq C m_{0}\left(\Lambda_{\ell}^{j}\right) .
$$

More generally, for any $k \in \mathbb{N}$, for any $x \in \Delta$ such that $F^{k}(x) \in \Lambda_{0}$,

$$
C^{-1} m_{0}\left(C_{k}(x)\right) \leq J F^{k}(x)^{-1} \leq C m_{0}\left(C_{k}(x)\right) .
$$

So $\mathrm{E}_{0}$ is well defined and acts continuously on the space $C_{u}(\Delta)$ of uniformly continuous and bounded functions on $\Delta$, it also acts on $L^{1}\left(m_{0}\right)$.

The following facts on $\mathrm{E}_{0}$ directly follow from the definitions:

- $\int \mathrm{E}_{0} f \cdot g d m_{0}=\int g \circ F \cdot f d m_{0}$ for all $f$ and $g$ in $C_{u}(\Delta)$ (i.e. $m_{0}$ is a conformal measure),

- a measure $\mu=h_{0} m_{0}, h_{0} \in L^{1}(m)$ is $F$ invariant if and only if $\mathrm{E}_{0} h_{0}=h_{0}$.

We expect that the mixing properties of an invariant measure absolutely continuous with respect to $m_{0}$ are related to the spectral properties of $\mathrm{E}_{0}$. To be more precise, let us assume that there exists a fixed point $h_{0} \in C_{u}(\Sigma)$ for $\mathrm{E}_{0}$ which is normalized $\left(m_{0}\left(h_{0}\right)=1\right)$ and let $\mu=h_{0} m_{0}$. For $f \in C_{u}(\Sigma)$ and $g \in L^{1}\left(m_{0}\right)$, the correlations of $f$ and $g$ measure the lack of independence between $f$ and $g \circ F^{n}$ with respect to the invariant measure $\mu$ : for $n \in \mathbb{N}$,

$$
c_{n}(f, g)=\left|\int f\left(g \circ F^{n}\right) d \mu-\int f d \mu \int g d \mu\right| .
$$

The measure $\mu$ is mixing if and only if the coefficients $c_{n}(f, g)$ go to zero for any $f \in C_{u}(\Sigma)$ and $g \in L^{1}\left(m_{0}\right)$. In this case, estimates on the speed of convergence to zero of $c_{n}(f, g)$ or, equivalently, estimates on the decay of correlations may lead to the Central Limit Theorem (see [Li2]) and to the determination of asymptotic laws for entrance times (see $[\mathrm{G}, \mathrm{S}$ and $[\mathrm{Sau}]$ ). The following trivial computation relates the decay of correlations to the asymptotic behavior of the iterates of $\mathrm{E}_{0}$ :

$$
c_{n}(f, g)=\left|\int\left[\mathrm{E}_{0}^{n}\left(f h_{0}\right)-h_{0} m_{0}\left(f h_{0}\right)\right] g d m_{0}\right|
$$


so that if $\mathrm{E}_{0}^{n} f \rightarrow h_{0} m_{0}(f)$ in some reasonable way, then $\mu$ is mixing and estimates on the speed of this convergence would lead to estimates on the decay of correlations.

We will denote by $L$ the space of functions that are uniformly Lipschitz (u.L.) and bounded on $\Delta$. That is, bounded functions $f$ satisfying the u.L. condition:

there exists $L(f)>0$, such that for $x$ and $y$ in the same $\Lambda_{\ell}$,

$$
|f(x)-f(y)| \leq L(f) d(x, y) .
$$

Let $\|f\|=\max \left(\|f\|_{\infty}, L(f)\right)$, this defines a norm on $L$ which turns $L$ into a Banach space.

1.1. Invariant measure and decay of correlations. Under some additional assumptions, we will prove existence and uniqueness on an $F$-invariant probability, absolutely continuous with respect to $m_{0}$. We will also give some estimations on the decay of correlations when this invariant measure is mixing.

Following $\mathrm{A}, \mathrm{D}, \mathrm{U}]$, we will say that the system $(\Delta, F)$ is irreducible if for all $i, j, \ell, \ell^{\prime}$, there exists an integer $n$ such that $F^{-n} \Lambda_{\ell}^{j} \cap \Lambda_{\ell^{\prime}}^{i} \neq \varnothing$; we will say that the system $(\Delta, F)$ is aperiodic if for all $i, j, \ell, \ell^{\prime}$, there exists an integer $N$ such that for all $n \geq N, F^{-n} \Lambda_{\ell}^{j} \cap \Lambda_{\ell^{\prime}}^{i} \neq \varnothing$. Following Markov chains and positive matrices methods ( $\mathrm{Se}]$ ), it may be proved that aperiodicity is equivalent to L.-S. Young's condition: g. c. d. $R=1$ ( $[\mathrm{Y} 2]$ ).

To study mixing properties of $\mathrm{E}_{0}$, we will need to deal with more general potentials.

Let $\Phi: \Delta \longrightarrow \mathbb{R}$ satisfy the uniformly locally Lipschitz (u.l.L.) condition:

there exists a constant $L>0$ such that, for all $x$ and $y$ in the same $\Lambda_{\ell}^{j}$,

$$
|\Phi(x)-\Phi(y)| \leq L d(x, y) .
$$

The associated transfer operator $\mathrm{t}_{\Phi}$ is well defined if

$$
\forall x \in \Delta, \sum_{F y=x} e^{\Phi(y)}<\infty .
$$

It is then defined by

$$
\mathrm{Ł}_{\Phi} f(x)=\sum_{F y=x} e^{\Phi(y)} f(y) .
$$

Under some general conditions, the operator $\mathrm{E}_{\Phi}$ admits a unique nonzero positive fixed point.

Theorem 1.2. Let $\Phi$ satisfy the u.l.L condition, have a conformal measure $m^{\prime}$ (i.e. $\left.m^{\prime}\left(E_{\Phi} f\right)=m^{\prime}(f) \forall f \in C_{u}(\Delta)\right)$, satisfy the "weak contribution of infinity" condition:

$$
\exists n_{0} / \forall n \geq n_{0} \sup _{x \in \Lambda_{n}}\left|E_{\Phi} \mathbf{1}(x)\right| \leq 1,
$$

and $\left\|E_{\Phi} \mathbf{1}\right\|_{\infty}<\infty$, then $E_{\Phi}$ admits a nonzero positive fixed point $h_{\Phi} \in L$.

If $(\Delta, F)$ is irreducible, then $h_{\Phi}>0$, unique up to a multiplicative factor and the invariant measure $\mu=h_{\Phi} m^{\prime}$ is ergodic.

If $(\Delta, F)$ is aperiodic, then $\mu$ is mixing. Moreover $\epsilon_{\Phi}^{n} f$ converges to $h_{\Phi} m^{\prime}(f)$ uniformly on each compact subset of $\Delta$ and in $L^{1}\left(m^{\prime}\right)$ for any $f \in C_{u}(\Delta)$.

Remark 1.3. The function $\Phi$ is not necessarily bounded but the hypothesis $\left\|\mathrm{E}_{\Phi} \mathbf{1}\right\|_{\infty}<\infty$ implies that the function $e^{\Phi}$ is bounded. 
The proof of Theorem 1.2 is now more or less standard. It follows arguments from [Sa] and [Ma1, Ma2] (see also Y Y2] for a proof without transfer operator). We will just sketch the different steps. We begin with the following lemma.

Lemma 1.4. Let $\Phi$ satisfy the hypothesis of Theorem 1.2, then there exists $M>0$ such that $\forall k \in \mathbb{N},\left\|E_{\Phi}^{k} \mathbf{1}\right\|_{\infty} \leq M$.

Proof. First, let us note that the u.l.L condition implies the following bounded distortion property: $\exists C>0$ such that $\forall x, y$ in the same $\Lambda_{\ell}$,

$$
\forall k \in \mathbb{N},\left|\frac{\mathrm{E}_{\Phi}^{k} \mathbf{1}(x)}{\mathrm{Ł}_{\Phi}^{k} \mathbf{1}(y)}-1\right| \leq C d(x, y) .
$$

Let $\Phi$ satisfy the "weak contribution of infinity condition". For any $k \in \mathbb{N}, n \in \mathbb{N}$ and $x \in \Lambda_{n}$, the bounded distortion property gives

$$
\begin{aligned}
\mathrm{E}_{\Phi}^{k} \mathbf{1}(x) & \leq C \frac{1}{m^{\prime}\left(\Lambda_{n}\right)} \int_{\Lambda_{n}} \mathrm{E}_{\Phi}^{k} \mathbf{1} d m^{\prime} \\
& \leq C \frac{1}{m^{\prime}\left(\Lambda_{n}\right)} \int_{\Delta} \mathrm{E}_{\Phi}^{k} \mathbf{1} d m^{\prime} \\
& =C \frac{1}{m^{\prime}\left(\Lambda_{n}\right)} \text { because } m^{\prime} \text { is a conformal probability. }
\end{aligned}
$$

So, $M^{\prime}>0$ exists such that $\sup _{x \in \Lambda_{n}} \mathrm{E}_{\Phi}^{k} \mathbf{1}(x) \leq M^{\prime}$ for all $k \in \mathbb{N}$ and for all $n \leq n_{0}$. It remains to control $\mathrm{E}_{\Phi}^{k} \mathbf{1}(x)$ for $k \in \mathbb{N}$ and $x \in \Lambda_{n}, n \geq n_{0}$. Let us denote by $M_{k}=\sup _{x \in \Delta} \mathrm{E}_{\Phi}^{k} \mathbf{1}(x)<\infty$ because of $\left\|\mathrm{E}^{k} \mathbf{1}\right\|<\infty$ for all $k$. Since $\Phi$ satisfies the weak contribution of infinity assumption, if $x$ belongs to $\Lambda_{n}, n \geq n_{0}$, then $\mathrm{E}_{\Phi} \mathbf{1}(x) \leq 1$ and

$$
\mathrm{E}_{\Phi}^{k+1} \mathbf{1}(x) \leq \mathrm{E}_{\Phi} \mathbf{1}(x) \cdot \sup _{\Delta} \mathrm{E}_{\Phi}^{k} \mathbf{1} \leq M_{k}
$$

So, $M_{k+1} \leq \max \left(M^{\prime}, M_{k}\right)$ which leads to $M_{k} \leq \max \left(M^{\prime}, 1\right)$ which concludes the proof.

Sketch of Proof of Theorem 1.2. From Lemma 1.4 there exists $M>0$ such that $\left\|\mathrm{E}_{\Phi}^{k} \mathbf{1}\right\|_{\infty} \leq M$ for all $k \in \mathbb{N}$. It is then easy to see that $\mathrm{E}_{\Phi}$ acts on $L$. Indeed, letting $f$ belong to $L$ we have the following inequality: $\forall k \in \mathbb{N}$

$$
\left|\mathrm{E}_{\Phi}^{k} f(x)-\mathrm{E}_{\Phi}^{k} f(y)\right| \leq d(x, y) \mathrm{Ct}\left[\|f\|_{\infty}+L(f)\right] \forall x, y \text { in the same floor. }
$$

Ascoli's theorem on separable spaces implies that there exists a sequence $n_{k}$ such that $Q_{n_{k}}=\frac{1}{n_{k}} \sum_{i=0}^{n_{k}-1} \mathrm{E}_{\Phi}^{i} \mathbf{1}$ converges, uniformly on compact subsets of $\Delta$ and in $L^{1}\left(m^{\prime}\right)$, to some limit $h_{\Phi} \in L$. Using the fact that $m^{\prime}$ is conformal, we get that this limit is nonzero (indeed, $m^{\prime}\left(h_{\Phi}\right)=1$ by Lebesgue's theorem) and satisfies $\mathrm{E}_{\Phi} h_{\Phi}=h_{\Phi}$. Let $\mu=h_{\Phi} m^{\prime}$; this is a $F$-invariant probability.

If $(\Delta, F)$ is irreducible, then $\mathrm{A}, \mathrm{D}, \mathrm{U}$ (Theorem 2.5) implies that $\mu=h m$ is ergodic and $h>0$.

Using A,D,U Theorem 3.2 (see also Young's arguments [Y2]), aperiodicity of the system implies that $(F, \mu)$ is exact. If $\mu$ is mixing, then $\mathrm{E}_{\Phi}^{k} f$ converges to $h_{\Phi} m^{\prime}(f)$ uniformly on each compact subset of $\Delta$ (any accumulation point of $\mathrm{E}_{\Phi}^{k} f$ must be $h_{\Phi} m^{\prime}(f)$ because of mixing).

Recall that $\mathrm{E}_{0}$ is the transfer operator associated to the potential $-\log J F$. 
Theorem 1.5. The operator $E_{0}$ admits a nonzero positive fixed point $h_{0} \in L$.

If $(\Delta, F)$ is irreducible, then $h_{0}>0$ is unique up to a multiplicative factor and the invariant measure $\mu=h_{0} m_{0}$ is ergodic.

If $(\Delta, F)$ is aperiodic, then $\mu$ is mixing. Moreover $E_{0}^{n} f$ converges to $h_{0} m_{0}(f)$ uniformly on each compact subset of $\Delta$ and in $L^{1}\left(m_{0}\right)$ for any $f \in C_{u}(\Delta)$.

For any increasing sequence $\left(v_{n}\right)_{n \in \mathbb{N}}$ such that $\sum_{\ell \in \mathbb{N}} m_{0}\left(\Lambda_{\ell}\right) \cdot v_{\ell}<\infty$, for any $f \in L$ and $g$ such that $g v \in L^{1}\left(m_{0}\right)$ (gv is defined by $g v(x)=g(x) v_{\ell}$ if $x$ belong to $\left.\Lambda_{\ell}\right)$, there exists $0<\gamma<1$ such that

$$
c_{n}(f, g) \leq \mathrm{Ct} \max \left[\left(v_{n}\right)^{-1}, \gamma^{n}\right]\|f\|\|g v\|_{1} .
$$

We also estimate the speed of convergence of $E_{0}^{n} f$ to $h_{0} m_{0}(f)$ in the uniform norm on each floor.

In particular,

- if there exist constants $0<e_{1}$ and $0<\theta<1$ such that for all $\ell \in \mathbb{N}, m\left(\Lambda_{\ell}\right) \leq$ $e_{1} \theta^{\ell}$, then there exists $0<\gamma<1$ such that for any $\theta^{\prime}<\theta$, for $g$ such that $\frac{g}{\theta} \in L^{1}(m)$ (where $\frac{g}{\theta^{\prime}}(x)=\frac{g(x)}{\theta^{\prime \ell}}$ for $\left.x \in \Lambda_{\ell}\right)$,

$$
c_{n}(f, g) \leq \operatorname{Ct}\left(\theta^{\prime}\right) \gamma^{n}\|f\|\left\|\frac{g}{\theta^{\prime}}\right\|_{1},
$$

- if there exist constants $0<e_{1}, 0<\theta<1$ and $0<\beta<1$ such that for all $\ell \in \mathbb{N}, m\left(\Lambda_{\ell}\right) \leq e_{1} \theta^{\ell^{\beta}}$, then for any $\beta^{\prime}<\beta$, let $v_{\ell}=\theta^{-\ell^{\beta^{\prime}}}$,

$$
c_{n}(f, g) \leq \mathrm{Ct}\left(\beta^{\prime}\right) \theta^{n^{\beta^{\prime}}}\|f\|\|g v\|_{1},
$$

- if there exist constants $0<e_{1}, \beta>1$ such that for all $\ell \in \mathbb{N}, m\left(\Lambda_{\ell}\right) \leq e_{1} \ell^{-\beta}$, then for any $\gamma>1$, let $v_{\ell}=\left[\frac{(\ln \ell)^{\gamma}}{\ell^{\beta-1}}\right]^{-1}$,

$$
c_{n}(f, g) \leq \operatorname{Ct}(\gamma) \frac{(\ln n)^{\gamma}}{n^{\beta-1}}\|f\|\|g v\|_{1} .
$$

Remark 1.6. On the one hand, L-S. Young obtained a decay of correlations in $O\left(n^{-(\beta-1)}\right)$ in the polynomial case above. Our result is a little bit slower in this case. Except for this, we rediscover her results. On the other hand, we get that the decay of correlations is of the form $c_{n}(f, g) \leq\left(v_{n}\right)^{-1} C(f, g)$ where the dependence of $C(f, g)$ on $g$ is given by $\|g v\|_{1}$. This kind of property is very useful in studying asymptotic laws of successive return times (see $[\mathrm{C}, \mathrm{G}, \mathrm{S}], \mathrm{G}, \mathrm{S}],[\mathrm{Sau},[\mathrm{P}]$ ) and was not obtained using coupling methods as in $\mathrm{Y} 2$.

Existence and mixing properties of an invariant measure absolutely continuous with respect to $m_{0}$ follow from Theorem 1.2, Remark that (1.1) and (BD) imply that the hypothesis of Lemma 1.4 is satisfied for $\Phi=-\log J F$ (obviously from definitions, $m_{0}$ is a conformal probability).

Our estimation of the decay of correlations is based on Birkhoff's cones and projective metrics. Let us briefly recall basic definitions and facts on this theory.

\section{BIRKHOFF'S CONES AND PROJECTIVE METRICS}

The theory of cones and projective metrics of G. Birkhoff [Bi1] is a powerful tool to study linear operators. P. Ferrero and B. Schmitt [F,S1 applied it to estimate the correlation decay for random dynamical systems. This strategy had been used by many authors. Let us mention C. Liverani [Li1] and M. Viana [V] for Anosov and Axiom A diffeomorphisms. They used Birkhoff cones to obtain exponential 
decay of correlations. In $\bar{K}, \mathrm{M}, \mathrm{S}$ the Birkhoff cones techniques were used in a different way to obtain sub-exponential decay of correlations. The way we use cone techniques to prove Theorem 1.5 follows some ideas of P. Ferrero and B. Schmitt $([\mathrm{F}, \mathrm{S} 2])$ and [Ma1, Ma2].

Let us recall definitions and properties of cones and projective metrics (see [Li1] for a more complete presentation).

Let $B$ be a vector space and $C \subset B$ a cone with the following properties.

- $C$ is convex,

- $C \cap-C=\{0\}$,

- if $\alpha_{n}$ is a sequence of real numbers such that $\alpha_{n} \rightarrow \alpha$ and $x-\alpha_{n} y \in C \forall n$ then $x-\alpha y \in C$. This property is called "integral closure".

For such a cone, the pseudo-metric $\theta_{C}$ on $C$ is defined in the following way. Let $x, y \in C$,

$$
\begin{aligned}
& \mu(x, y)=\inf \{\beta>0 \text { such that } \beta x-y \in C\}, \\
& l(x, y)=\sup \{\alpha>0 \text { such that } y-\alpha x \in C\},
\end{aligned}
$$

with the convention: $\mu(x, y)=\infty$ and $l(x, y)=0$ if the corresponding sets are empty. Let $\theta_{C}(x, y)=\log \frac{\mu}{l}$. $\theta_{C}$ is called pseudo-metric because it is not necessarily finite. Moreover, it is a projective pseudo-metric: if $x$ and $x_{1}$ are proportional, then for any $y \in C, \theta_{C}(x, y)=\theta_{C}\left(x_{1}, y\right)$.

The following two results reveal the usefulness of projective metrics. Let $C$ and $C^{\prime}$ be two cones, $P$ a linear operator $P: C \rightarrow C^{\prime}$. Let $\Delta$ denote the diameter of $P C$ in $C^{\prime}$ :

$$
\Delta=\sup _{f, g \in C} \theta_{C^{\prime}}(P f, P g)
$$

Theorem 2.1 (Bi1]). For any $f, g$ in $C$, we have

$$
\theta_{C^{\prime}}(P f, P g) \leq \tanh \left(\frac{\Delta}{4}\right) \theta_{C}(f, g)
$$

This theorem implies that $P: C \rightarrow C^{\prime}$ is always a contraction (in the wide sense) for the projective metrics. If $\Delta<\infty$, then it is a strict contraction.

The following result relies the metric $\theta_{C}$ to certain norms on $B$. A norm \|\| on $B$ is a norm adapted to $C$ if for $f$ and $g$ in $B$ such that if $f+g$ belongs to $C$ and $f-g$ belongs to $C$, then $\|g\| \leq\|f\| . \rho$ is a homogeneous form adapted to $C$ if $\rho$ maps $C$ to $\mathbb{R}^{+}$, for any $l>0$ and $f \in C, \rho(l f)=l \rho(f)$ and if $f-g \in C$ implies $\rho(g) \leq \rho(f)$.

Theorem 2.2 ([Bi1], [Li1]). Let $C$ be a cone, let \|\| and $\rho$ be adapted to $C$. For any $f$ and $g$ in $C$ such that $\rho(f)=\rho(g) \neq 0$ we have

$$
\|f-g\| \leq\left(e^{\theta(f, g)}-1\right) \min (\|f\|,\|g\|) .
$$

\section{Proof of Theorem 1.5}

We will now prove Theorem 1.5. Let us begin with the following remark.

Remark 3.1. Let $v=\left(v_{\ell}\right)_{\ell \in \mathbb{N}}$ be an increasing sequence of positive numbers such that

$$
\sum_{\ell \in \mathbb{N}} m_{0}\left(\Lambda_{\ell}\right) \cdot v_{\ell}<\infty
$$


We will consider such a $v$ as a function on $\Delta: v(x)=v_{\ell}$ if $x$ belongs to $\Lambda_{\ell}$. Let $\mathrm{E}$ be the transfer operator associated to the change of potential $v$ : $\mathrm{\complement} f=v^{-1} \mathrm{~L}_{0}(f v)$. The measure $m=v m_{0}$ is conformal for $\mathrm{E}$ and it follows easily from (1.1) and Lemma 1.4 that $\sup _{k \in \mathbb{N}}\left\|\mathrm{E}^{k} \mathbf{1}\right\|_{\infty}<\infty$. Moreover, $h=\frac{h_{0}}{v}$ is an eigenvector for $\mathrm{E}$. Theorem 1.2 implies that for any $f \in C_{u}(\Delta)$,

$$
\mathrm{E}^{k} f \longrightarrow h m(f) \text { uniformly on each compact set. }
$$

Throughout this section, we fix a strictly increasing sequence $v=\left(v_{\ell}\right)_{\ell \in \mathbb{N}}$ such that $m(\Delta)<\infty$ and the sequence $\left(\frac{v_{\ell}}{v_{\ell+1}}\right)_{\ell \in \mathbb{N}}$ is also increasing. We will assume that $m$ and $h$ are normalized $(m(\Delta)=1$ and $m(h)=1)$. We will work with the transfer operator $\mathrm{E}$.

As usual in these topics, the main tool is a "Lasota-Yorke" type inequality. Before proving it, let us study the distance on $\Delta$ a bit.

3.1. Some properties of the distance on $\Delta$. Let us consider the following function on $\Delta: \rho(x)=\beta$ if $x$ belongs to $\Lambda_{0}$ and $\rho(x)=1$ otherwise. For $k \in \mathbb{N}$, we will denote by $\rho^{(k)}(x)=\prod_{i=0}^{k} \rho\left(F^{i} x\right)$. The relation between $\rho^{(k)}$ and the distance on $\Delta$ is the following:

if $x$ and $y$ are in $\Lambda_{0}$, their preimages by $F^{k}$ are paired. If $x^{\prime}$ is a preimage of $x$, we will denote by $y^{\prime}$ the preimage of $y$ which belongs to $C_{k}\left(x^{\prime}\right)$. We have

$$
d\left(x^{\prime}, y^{\prime}\right)=\rho^{(k)}\left(x^{\prime}\right) \cdot d(x, y) .
$$

The following lemma proves that $\rho^{(k)}(x)$ is almost exponential.

Lemma 3.2. There exists $0<\gamma<1$ such that for all $\eta>0$, there exists $\Omega_{\eta} \subset \Delta$ of $m$ measure greater than $1-\eta$ and $k_{0}(\eta)$ such that for all $k \geq k_{0}$ and $x \in \Omega_{\eta}$, $\rho^{(k)}(x) \leq \gamma^{k}$.

Proof. This is an easy consequence of Birkhoff's ergodic theorem and Egoroff's theorem applied to $\log \rho$.

Remark 3.3. A finer analysis on $\rho^{(k)}$ should allow one to avoid the use of Birkhoff's and Egoroff's theorems and so give a constructive bound for $k_{0}$.

3.2. A Lasota-Yorke type inequality. We are now in position to prove a LasotaYorke type inequality.

Lemma 3.4. For any $\varepsilon>0$, there exists $C>0, N \in \mathbb{N}$ and $k_{0} \in \mathbb{N}$ such that for all $k>k_{0}$, for all $f \in L$, we have

1. for $\ell \geq k, x$ and $y$ in $\Lambda_{\ell}$

$$
\left|E^{k} f(x)-E^{k} f(y)\right| \leq \frac{v_{\ell-k}}{v_{\ell}} d(x, y) L(f),
$$

2. for $N \leq \ell<k, x$ and $y$ in $\Lambda_{\ell}$

$$
\left|E^{k} f(x)-E^{k} f(y)\right| \leq \varepsilon d(x, y)[L(f)+\sup |f|],
$$

3. for $\ell<N, x$ and $y$ in $\Lambda_{\ell}$

$$
\left|E^{k} f(x)-E^{k} f(y)\right| \leq d(x, y)[\varepsilon L(f)+C \sup |f|] .
$$


Proof. Let $f$ belong to $L$. Item 1 directly follows from the definition of $\mathrm{E}$ and $d(x, y)$.

Let $x$ and $y$ be in $\Lambda_{\ell}, \ell<k$, and let us denote by $x^{0} \in \Lambda_{0}$ (resp. $\left.y^{0}\right)$ the preimage of $x$ (resp. $y$ ) by $F^{k-\ell}$. We have

$$
\left|\mathrm{E}^{k} f(x)-\mathrm{E}^{k} f(y)\right|=\frac{v_{0}}{v_{\ell}}\left|\mathrm{E}^{k-\ell} f\left(x^{0}\right)-\mathrm{E}^{k-\ell} f\left(y^{0}\right)\right| .
$$

Let us consider $x$ and $y$ in $\Lambda_{0}$; their paired preimages will be denoted by $x^{\prime}$ and $y^{\prime}$.

$$
\begin{aligned}
& \left|\mathrm{E}^{k} f(x)-\mathrm{七}^{k} f(y)\right|=\frac{1}{v_{0}}\left|\sum_{F^{k} x^{\prime}=x} \frac{v\left(x^{\prime}\right)}{J F^{k}\left(x^{\prime}\right)} f\left(x^{\prime}\right)-\frac{v\left(y^{\prime}\right)}{J F^{k}\left(y^{\prime}\right)} f\left(y^{\prime}\right)\right| \\
& \quad \leq \frac{1}{v_{0}} \sum_{F^{k} x^{\prime}=x} \frac{v\left(x^{\prime}\right)}{J F^{k}\left(x^{\prime}\right)}\left|f\left(x^{\prime}\right)-f\left(y^{\prime}\right)\right| \\
& \quad+\frac{1}{v_{0}} \sum_{F^{k} x^{\prime}=x} \frac{v\left(x^{\prime}\right)}{J F^{k}\left(x^{\prime}\right)}\left|f\left(y^{\prime}\right)\right|\left|\frac{J F^{k}\left(x^{\prime}\right)}{J F^{k}\left(y^{\prime}\right)}-1\right| \\
& \leq L(f) d(x, y) \underbrace{\sum_{F^{k} x^{\prime}=x} v\left(x^{\prime}\right) J F^{k}\left(x^{\prime}\right)^{-1} \rho^{(k)}\left(x^{\prime}\right)}_{(1)} \\
& \quad+C d(x, y) \sup |f| \underbrace{\sum_{F^{k} x^{\prime}=x} v\left(x^{\prime}\right) J F^{k}\left(x^{\prime}\right)^{-1}}_{(2)} .
\end{aligned}
$$

The term (2) is bounded above by $\left\|\mathrm{E}^{k} \mathbf{1}\right\|_{\infty} \leq M$.

Let us turn our attention to term (1). Let $\eta>0$ to be fixed later, let $\Omega_{\eta}$ be given by Lemma 3.2 and assume $k \geq k_{0}(\eta)$. Recall (1.2): for $z$ such that $F^{k}(z) \in \Lambda_{0}$, $J F^{k}(z)^{-1} \leq C m_{0}\left(C_{k}(z)\right)$ and remark that $\rho^{(k)}$ is constant on each $k$-cylinder. Using Lemma 3.2 we get

$$
\begin{aligned}
(1) & \leq \sum_{\substack{F^{k} x^{\prime}=x \\
\rho^{(k)}\left(x^{\prime}\right) \leq \gamma^{k}}} v\left(x^{\prime}\right) J F^{k}\left(x^{\prime}\right)^{-1} \rho^{(k)}\left(x^{\prime}\right)+\sum_{\substack{F^{k} x^{\prime}=x \\
x^{\prime} \in\left(\Omega_{\eta}\right)^{c}}} v\left(x^{\prime}\right) J F^{k}\left(x^{\prime}\right)^{-1} \rho^{(k)}\left(x^{\prime}\right) \\
& \leq \gamma^{k} M+C m\left(\Omega_{\eta}^{c}\right) \leq C\left(\gamma^{k}+\eta\right) .
\end{aligned}
$$

We have proved that for $x$ and $y$ in $\Lambda_{0}$, for any $\eta>0$, provided $k$ is large enough,

$$
\left|\mathrm{七}^{k} f(x)-\mathrm{E}^{k} f(y)\right| \leq C d(x, y)\left[\left(\gamma^{k}+\eta\right) L(f)+\sup |f|\right] .
$$

Remark that we have proved also the following weak inequality for all $k$ :

$$
\left|\mathrm{七}^{k} f(x)-\mathrm{七}^{k} f(y)\right| \leq C d(x, y)[L(f)+\sup |f|]
$$

Let $\varepsilon$ be fixed and choose :

- $\eta$ such that $C \eta<\varepsilon / 2$, let $k_{1}=k_{0}(\eta)$ be given by Lemma 3.2 ,

- $N>0$ such that $C \frac{v_{0}}{v_{N}}<\varepsilon$,

- $k_{0}>k_{1}+N$ and for $k>k_{0}-N, C \gamma^{k}<\varepsilon / 2$.

Let $k>k_{0}$ and $N \leq \ell<k$, the choices above and (3.4) give

$$
\left|\mathrm{E}^{k} f(x)-\mathrm{七}^{k} f(y)\right| \leq \frac{v_{0}}{v_{\ell}} C d(x, y)[L(f)+\sup |f|] \leq \varepsilon d(x, y)[L(f)+\sup |f|] .
$$


Letting $\ell \leq N$, we have

$$
\begin{aligned}
\left|\mathrm{E}^{k} f(x)-\mathrm{E}^{k} f(y)\right| & \leq \frac{v_{0}}{v_{\ell}} C d(x, y)\left[\left(\gamma^{k-\ell}+\eta\right) L(f)+\sup |f|\right] \\
& \leq d(x, y)\left[\varepsilon L(f)+C\|f\|_{\infty}\right] .
\end{aligned}
$$

Remark 3.5. This is clear from the proof of Lemma 3.4 that trying to get a LasotaYorke inequality for the transfer operator $\mathrm{Ł}_{0}$ instead of $\mathrm{E}$ would lead to some "weak Lasota-Yorke inequality", i.e. the coefficient in front of $L(f)$ would not be strictly smaller than 1.

3.3. Construction of cones. As stated in the introduction, to prove Theorem 1.5, we are going to use cone techniques. Let us explain a bit how to construct the cones and how sub-exponential decay of correlations may be obtained.

We start by recalling the classical way of using cones (see [F,S1] and [Li1] for details). To get exponential decay of correlations, it is sufficient to find a cone $C$ and an integer $k$ such that $\mathrm{E}^{k}$ maps $C$ into itself and the diameter $d_{0}$ of $\mathrm{E}^{k} C$ into $C$ is finite. If the fixed point $h$ of $\mathrm{E}$ belongs to $C$ then Theorem 2.1 gives, for any integer $j$,

$$
\theta_{C}\left(\mathrm{E}^{k j} f, h\right) \leq \gamma^{j-1} d_{0} \text { where } \gamma=\tanh \frac{d_{0}}{4}<1 .
$$

Then Theorem 2.2 gives for $f \in C$, that $\left\|\mathrm{E}^{p} f-h m(f)\right\|$ goes to zero exponentially fast for \|\| an adapted norm, provided $f \mapsto m(f)$ is adapted. Then one has to extend this result from the cone to a Banach space.

The starting point of the construction of cones is usually a Lasota-Yorke inequality. Looking at Lemma 3.4, one sees that in our context the Lasota-Yorke inequality depends on the floors of the tower, it is not uniform unless if $v_{\ell}=\frac{\mathrm{Ct}}{\theta^{\ell}}$ for some $0<\theta<1$, this is equivalent to the fact that $m_{0}\left(\Lambda_{\ell}\right) \leq \mathrm{Ct} \theta^{\prime \ell}$ for some $0<\theta^{\prime}<\theta$. In general for fixed $k, \lim \sup _{\ell \rightarrow \infty} \frac{v_{\ell-k}}{v_{\ell}}=1$. This is the reason why we have to deal with a sequence of cones instead of a single one and why we get sub-exponential decay of correlations.

Roughly speaking, for some fixed integer $k$ and any integer $j$, we will consider a cone $C_{j}$ of functions $f$ for which we control the Lipschitz constant on the first $k j$ floors (see condition 2 below). Thanks to the definition of $\mathrm{E}$ and to Lemma 3.4 if $f$ belongs to $C_{j}$ then we will control the Lipschitz constant of $\mathrm{E}^{k} f$ on the first $k(j+1)$ floors and the control is improved by a factor $\frac{v_{k j}}{v_{k(j+1)}}<1$. This should imply that $\mathrm{E}^{k}$ maps $C_{j}$ into $C_{j+1}$ with finite diameter $d_{j}$ such that $\tanh \frac{d_{j}}{4} \leq \frac{v_{k j}}{v_{k(j+1)}}$. Then, using Theorems 2.1 and 2.2, we get that for $f$ in $C_{0}, \mathrm{七}^{k j} f$ goes to $m(f) h$ at rate $\frac{v_{0}}{v_{k j}}$ in any adapted norm, provided that $h$ belongs to all the cones $C_{j}$ and that $f \mapsto m(f)$ is adapted. This is the philosophy of the construction.

Unfortunately, things are not so simple. Firstly, to control $\mathrm{E}^{k} f$ on $\Lambda_{0}, \ldots, \Lambda_{k}$, we need to control $f$ on all the floors (not only the first $k j$ 's) because of the definition of $\mathrm{E}$.

Secondly, to ensure that a cone $C$ satisfies properties of section 2 and more specifically the condition $C \cap-C=\{0\}$, some positivity for the functions in the cone is needed. Also, if $C \subset\{f \geq 0\}=: C_{+}$, then for any $f, g$ in $C, \theta_{C}(f, g) \geq \theta_{C_{+}}(f, g)$ 
(use Theorem 2.1 with $P=I d$ ) and

$$
\theta_{C_{+}}(f, g)=\frac{\sup f}{\inf f} \cdot \frac{\sup g}{\inf g} .
$$

As already explained, we want the invariant density $h$ to belong to the cones. We know that for $x \in \Lambda_{\ell}, h(x)=\frac{h_{0}(x)}{v_{\ell}}$ where $h_{0}$ is the fixed point of $\mathrm{E}_{0}$ which is bounded by Theorem 1.5 Thus, there is no hope of controlling the quantity $\frac{\sup h}{\inf h}$.

These two problems are solved by considering a finite partition of $\Delta$ which is decomposed into finitely many $s$-cylinders (the "compact" part) and the complement of the union of these $s$-cylinders (the "noncompact" part). Then we require the positivity of some kind of conditional expectation of $f$ with respect to this finite partition (see condition 1 below). This together with the control of the Lipschitz constant on the low floors leads to a good control of $f$ on the atoms of the compact part. Then, we require another kind of control on the noncompact part (see condition 3 and 4 below).

Let us introduce the finite partition of $\Delta$. Let $\eta$ and $t$ be given, let $s$ be greater than $k_{0}(\eta)$ given by Lemma 3.2. These three parameters are used to control the noncompact part of $\Delta$. Let $\mathcal{P}$ be the finite partition $\mathcal{P}=\mathcal{P}_{1} \cup \mathcal{P}_{2}$ defined by:

- $\mathcal{P}_{2}=\left\{\bigcup_{R(j) \geq t} \bigcup_{\ell \in \mathbb{N}} \Lambda_{\ell}^{j} \cup\left\{x / \rho^{(s)}(x)>\gamma^{s}\right\}\right\}=:\left\{P_{2}\right\}$,

- $\mathcal{P}_{1}$ is the partition of $P_{2}^{c}$ into $s$-cylinders.

Because of Lemma 3.2, the $m$ measure of $P_{2}$ is as small as we want, provided $t$ is large enough and $\eta$ is small enough:

$$
m\left(P_{2}\right) \leq m\left(\bigcup_{R(j) \geq t} \bigcup_{\ell \in \mathbb{N}} \Lambda_{\ell}^{j}\right)+\eta .
$$

We will denote by $D_{1}$ the diameter of $\mathcal{P}_{1}$ (which is smaller than $\gamma^{s}$ by definition) and by $D_{2}$ the $m$ measure of $P_{2}$.

Theorem 1.2 implies the following mixing property: for $\alpha<1<\alpha^{\prime}$ there exists $q_{0}$ such that for any integer $k$ greater than $q_{0}$, for $P$ and $P^{\prime}$ elements of $\mathcal{P}$,

$$
\alpha<\frac{m\left(P \cap F^{-k} P^{\prime}\right)}{m(P) \mu\left(P^{\prime}\right)}<\alpha^{\prime} .
$$

Let $a, b, c, d$ be positive numbers and $k$ an integer to be chosen later. For any $j \in \mathbb{N}, \mathcal{C}(a, b, c, d, j)$ is the cone of functions in $L$ such that:

1. $0 \leq \frac{1}{\mu(P)} \int_{P} f d m \leq a \int f d m \forall P \in \mathcal{P}$,

2. for $\ell \leq j k$, for any elements $x$ and $y$ of $\Lambda_{l}$,

$$
|f(x)-f(y)| \leq d(x, y) b \int f d m,
$$

3. for $\ell \leq j k$, for any element $x$ of $\Lambda_{l} \cap P_{2},|f(x)| \leq c \int f d m$,

4. for $\ell>j k,|f(x)| \leq \frac{v_{k j}}{v_{\ell}} d \int f d m$ and

$$
|f(x)-f(y)| \leq \frac{v_{k j}}{v_{\ell}} d(x, y) d \int f d m \text {, for all } x, y \in \Lambda_{\ell} .
$$


Note that the cone also depends on $s, t, k, \eta$. We will choose $a, b, c, d, k, \eta, s$ and $t$ in such a way that $\mathrm{E}^{k}$ is a contraction from $\mathcal{C}(a, b, c, j)$ to $\mathcal{C}(a, b, c, d, j+1)$. Let us denote by $\delta_{j}=\frac{v_{k j}}{v_{k(j+1)}}<1$.

Remark 3.6. It will be clear from the proofs that if there exists $0<\beta<1$ such that $0<\delta_{j}<\beta$ then condition 4 in the definition of the cone is useless. This means that it is possible to work with the cone $\mathcal{C}(a, b, c)$ of functions $f$ such that:

$$
\begin{aligned}
& \text { 1. } 0 \leq \frac{1}{\mu(P)} \int_{P} f d m \leq a \int f d m \forall P \in \mathcal{P}, \\
& \text { 2. } L(f) \leq b \int f d m, \\
& \text { 3. for } x \in \Lambda_{\ell} \cap P_{2}|f(x)| \leq c \int f d m .
\end{aligned}
$$

Proposition 3.7. For any $0<\sigma<\delta_{j}$, for any positive numbers $a_{0}, b_{0}, c_{0}, d_{0}$, there exist an integer $k_{0}$, positive numbers $a \geq a_{0}, b \geq b_{0}, c \geq c_{0}, d \geq d_{0}$, and integers $s$ and $t$ such that for all $k>k_{0}$,

$$
E^{k} \mathcal{C}(a, b, c, d, j) \subset \mathcal{C}\left(\sigma a, \delta_{j} b, \delta_{j} c, \delta_{j} d, j+1\right) .
$$

Moreover, for $f \in E^{k} \mathcal{C}(a, b, c, d, j)$, we have

$$
\frac{1}{\mu(P)} \int_{P} f d m \geq A \text { for all } P \in \mathcal{P},
$$

where $A$ can be chosen arbitrarily provided it is strictly smaller than $1 / 2$.

Remark 3.8. In fact, it is clear from the proof that by modifying slightly the argument the constant $A$ above may be chosen arbitrarily close to 1 . This may be important to optimize the rate of convergence in the exponential case.

Before proving Proposition 3.7, let us explain where the contraction of each of the four conditions comes,

- the contraction of condition 1 will follow from the mixing and the contraction rate $\sigma$ may be obtained as close to 0 as we wish, provided $k$ is large enough,

- the contraction of condition 2 will follow from the Lasota-Yorke inequality,

- the contraction of condition 3 will follow mainly from the definition of $\mathrm{E}$ : for $\ell \geq k, \mathrm{E}^{k} f(x)=\frac{v_{\ell-k}}{v_{\ell}} f\left(x^{\prime}\right)$ for $x \in \Lambda_{\ell}$ and $F\left(x^{\prime}\right)=x, x^{\prime} \in \Lambda_{\ell-k}$,

- the contraction of condition 4 follows directly from the definition of $\mathrm{E}$.

We begin the proof of Proposition [3.7] with the following lemma which gives a control on the sup. of functions in $\mathcal{C}(a, b, c, d, j)$. When there is no ambiguity, we will forget about the dependence on $a, b, c, d$ and $j$ and write $\mathcal{C}$ instead of $\mathcal{C}(a, b, c, d, j)$ or $\mathcal{C}(j)$ if we wish to emphasize the dependence on $j$.

Lemma 3.9. For any $f \in \mathcal{C}$, for $\ell \in \mathbb{N}$ and $P \in \mathcal{P}_{1}$, for $x$ in $\Lambda_{\ell} \cap P$ we have

$$
\begin{aligned}
& |f(x)| \leq \frac{1}{m(P)} \int_{P} f d m+\max [b, d]|P| \int f d m, \\
& |f(x)| \leq\left[D a+D_{1} \max [b, d]\right] \int f d m .
\end{aligned}
$$


(where $\left.D=\sup _{P \in \mathcal{P}} \frac{\mu(P)}{m(P)}\right)$. For any $f \in \mathcal{C}$, for $x \in P_{2} \cap \Lambda_{\ell}, \ell \in \mathbb{N}$,

$$
\begin{aligned}
& f(x) \geq \frac{1}{m\left(P_{2}\right)} \int_{P_{2}} f d m-2 \max [c, d] \int f d m, \\
& f(x) \leq \max [c, d] \int f d m .
\end{aligned}
$$

Proof. Let $f$ belong to $\mathcal{C}$. For $x \in P \in \mathcal{P}_{1}$, equations (3.6) and (3.7) for $\ell \leq k j$ directly follow from definitions. Just write that for $x$ and $y$ in $P \in \mathcal{P}$,

$$
f(y)-L(f) d(x, y) \leq f(x) \leq f(y)+L(f) d(x, y)
$$

and integrate these inequalities on $P$ with respect to $m$. Let $x, y \in P_{2} \cap \Lambda_{\ell}$ with $\ell \leq k j$,

$$
f(y)-2 \sup _{\Lambda_{\ell} \cap P_{2}}|f| \leq f(x) \leq \sup _{\Lambda_{\ell} \cap P_{2}}|f|
$$

this leads to 3.8 for $\ell \leq k j$. Inequalities for $\ell>k j$ follow from 4 in the definition of the cone.

Let us begin with the proof of Proposition 3.7

Proof of Proposition 3.7. We are going to check how the four conditions in the definition of $\mathcal{C}$ evolve under the action of $\mathrm{E}^{k}$. First of all, condition 4 of the cone is very easily checked: if $x \in \Lambda_{\ell}, \ell>k(j+1)$, then $\mathrm{E}^{k} f(x)=\frac{v_{\ell-k}}{v_{\ell}} f\left(x^{\prime}\right)$, with $x^{\prime} \in \Lambda_{\ell-k}$ so $\left|\mathrm{E}^{k} f(x)\right|$ and $\left|\mathrm{E}^{k} f(x)-\mathrm{七}^{k} f(y)\right|$ are less than $\frac{v_{k(j+1)}}{v_{\ell}} \delta_{j} d \int f d m$, for $x$ and $y$ in $\Lambda_{\ell}, \ell>k j$. It just remains to check the first three conditions. Assume that $d$ is fixed, $b$ and $c$ will be chosen to have $d \leq \min [b, c]$.

We take $f \in \mathcal{C}$. We fix $0<\sigma<\delta_{j}$ and $\varepsilon<\sigma, \alpha<1<\alpha^{\prime}$ and $q_{0} \in \mathbb{N}$ such that (3.5) is satisfied for $k>q_{0}$. In what follows, $k$ is greater than $q_{0}$.

The following remark will be used many times in the proof: if $f \in \mathcal{C}$, then since we choose $d \leq \max (b, c)$ we have that $\sup |f| \leq c \int f d m$ and $L(f) \leq b \int f d m$ (use also that if $\ell>k j$, then $\frac{v_{k j}}{v_{\ell}} \leq 1$ ).

Condition 1. Let $P \in \mathcal{P}$,

$$
\begin{aligned}
\frac{1}{\mu(P)} \int_{P} \mathrm{E}^{k} f d m & =\frac{1}{\mu(P)} \int_{F^{-k} P} f d m=\sum_{P^{\prime} \in \mathcal{P}} \frac{1}{\mu(P)} \int_{P^{\prime} \cap F^{-k} P} f d m \\
& =\sum_{P^{\prime} \in \mathcal{P}_{1}} \frac{1}{\mu(P)} \int_{P^{\prime} \cap F^{-k} P} f d m+\frac{1}{\mu(P)} \int_{P_{2} \cap F^{-k} P} f d m .
\end{aligned}
$$

Using Lemma 3.9, we get for $P^{\prime} \in \mathcal{P}_{1}$ :

$$
\begin{aligned}
& \quad \frac{m\left(F^{-k} P \cap P^{\prime}\right)}{\mu(P) m\left(P^{\prime}\right)}\left[\int_{P^{\prime}} f d \mu-m\left(P^{\prime}\right) D_{1} b \int f d m\right] \leq \frac{1}{\mu(P)} \int_{P^{\prime} \cap F^{-k} P} f d m \\
& \text { and } \frac{1}{\mu(P)} \int_{P^{\prime} \cap F^{-k} P} f d m \leq \frac{m\left(F^{-k} P \cap P^{\prime}\right)}{\mu(P) m\left(P^{\prime}\right)}\left[\int_{P^{\prime}} f d \mu+m\left(P^{\prime}\right) D_{1} b \int f d m\right],
\end{aligned}
$$


using (3.5), this leads to

$$
\begin{aligned}
\alpha\left[\int_{P^{\prime}} f d m-\alpha^{\prime} b D_{1} m\left(P^{\prime}\right) \int f d m\right] & \leq \frac{1}{\mu(P)} \int_{P^{\prime} \cap F^{-k} P} f d m \\
\text { and } \frac{1}{\mu(P)} \int_{P^{\prime} \cap F^{-k} P} f d m & \leq \alpha^{\prime}\left[\int_{P^{\prime}} f d m+b D_{1} m\left(P^{\prime}\right) \int f d m\right] .
\end{aligned}
$$

For $P_{2}$, we get:

$$
\alpha \int_{P_{2}} f d m-2 c D_{2} \alpha^{\prime} \int f d m \leq \frac{1}{\mu(P)} \int_{P_{2} \cap F^{-k} P} f d m \leq \alpha^{\prime} c D_{2} \int f d m .
$$

Summing these inequalities over $P \in P_{1}$ and $P_{2}$, we get (one has to notice that $\left.m\left(\mathbf{1}_{P_{2}} \cdot f\right) \geq 0\right)$ :

$$
\begin{aligned}
& {\left[\alpha-\alpha^{\prime} D_{1} b-2 \alpha^{\prime} c D_{2}\right] \int f d m \leq \frac{1}{\mu(P)} \int_{P} \mathrm{E}^{k} f d m} \\
& \quad \text { and } \frac{1}{\mu(P)} \int_{P} \mathrm{E}^{k} f \leq\left[1+D_{1} b+c D_{2}\right] \alpha^{\prime} \int f d m
\end{aligned}
$$

Condition 2. Fix $\varepsilon>0$.

Let $x$ and $y$ belong to $\Lambda_{\ell}, \ell \leq k(j+1)$, using the fact that if $x \in \Lambda_{\ell}, \ell \leq k(j+1)$, then $F^{k} x^{\prime}=x$ with $x^{\prime} \in \Lambda_{\ell-k}$ and Lemma 3.4 we find $k_{0}$ and $N \in \mathbb{N}$ such that for $k>k_{0}$,

$$
\begin{aligned}
\left|\mathrm{E}^{k} f(x)-\mathrm{E}^{k} f(y)\right| & \leq \frac{v_{\ell-k}}{v_{\ell}} d(x, y) b \int f d m \text { if } \ell \geq k \\
& \leq \varepsilon d(x, y)\left[b \int f d m+\sup |f|\right] \text { if } N \leq \ell<k \\
& \leq d(x, y)\left[\varepsilon b \int f d m+C \sup |f|\right] \text { otherwise. }
\end{aligned}
$$

Lemma 3.9 gives $\sup |f| \leq \max \left[c, b D_{1}+a D\right] \int f d m$ so we get

$$
\begin{aligned}
& \left|\mathrm{E}^{k} f(x)-\mathrm{E}^{k} f(y)\right| \leq \frac{v_{\ell-k}}{v_{\ell}} d(x, y) b \int f d m \text { if } \ell \geq k \\
& \quad \leq \varepsilon d(x, y) \int f d m\left[b+\max \left(c, b D_{1}+a D\right)\right] \text { if } N \leq \ell<k \\
& \quad \leq d(x, y) \int f d m\left[\varepsilon b+C \max \left(c, b D_{1}+a D\right)\right] \text { otherwise. }
\end{aligned}
$$

Condition 3. Let $x \in \Lambda_{\ell} \cap P_{2}$ with $\ell \leq k(j+1)$, if $\ell \geq k$, we have

$$
\left|\mathrm{E}^{k} f(x)\right| \leq \frac{v_{\ell-k}}{v_{\ell}} c \int f d m .
$$

Let $\ell>k$, we denote by $x^{0}$ the preimage of $x$ by $F^{\ell}, x^{0} \in \Lambda_{0}$,

$$
\mathrm{E}^{k} f(x)=\frac{v_{0}}{v_{\ell}} \mathrm{Ł}^{k-\ell} f\left(x^{0}\right) .
$$


For $x \in \Lambda_{0}$ and $q \in \mathbb{N}$,

$$
\begin{aligned}
\left|\mathrm{E}^{q} f(x)\right| & \leq \sum_{\substack{F^{q} x^{\prime}=x \\
x^{\prime} \in P_{2}}} \frac{v\left(x^{\prime}\right)}{J F^{q}\left(x^{\prime}\right)} f\left(x^{\prime}\right)+\sum_{\substack{F^{q} x^{\prime}=x \\
x^{\prime} \notin P_{2}}} \frac{v\left(x^{\prime}\right)}{J F^{q}(x)} f\left(x^{\prime}\right) \\
& \leq c \int f d m C \sum_{\substack{F^{q} x^{\prime}=x \\
x^{\prime} \in P_{2}}} m_{0}\left(C_{q}\left(x^{\prime}\right)\right) v\left(x^{\prime}\right)+\int f d m\left[b D_{1}+a D\right] \text { using (3.7). }
\end{aligned}
$$

So we have for $x \in \Lambda_{\ell}, \ell<k$,

$$
\left|\mathrm{E}^{k} f(x)\right| \leq\left[c C D_{2}+b D_{1}+a D\right] \int f d m .
$$

We are now in position to choose the parameters. Choose

- $\sigma>\varepsilon$,

- $d>0$,

- $a>\frac{\alpha^{\prime}+3 / 8 \alpha}{\sigma}$,

- $c>\frac{1+a D}{\sigma-\varepsilon}, c \geq d$

- $b>\frac{C c}{\sigma-\varepsilon}, b \geq d$,

- $\eta$ and $t$ such that $2 \alpha^{\prime} c D_{2}<\alpha / 4$, and $C D_{2}<\varepsilon$,

- $s$ such that $s>k_{0}(\eta)\left(k_{0}(\eta)\right.$ is given by Lemma 3.2 and $\alpha^{\prime} c D_{1}=\alpha^{\prime} c \gamma^{s}<\alpha / 4$,

- $k>k_{0}(\eta), k>k_{0}(\varepsilon)$ given by Lemma 3.4 and $k>q_{0}$ given by (3.5).

With these choices, we have: for any $P \in \mathcal{P}$,

$$
\begin{aligned}
& \frac{\alpha}{2} \int f d m \leq \frac{1}{\mu(P)} \int_{P} \mathrm{E}^{k} f d m \leq\left(\alpha^{\prime}+3 / 8 \alpha\right) \int f d m \text { by (3.9), } \\
& \frac{\alpha}{2} \int f d m \leq \frac{1}{\mu(P)} \int_{P} \mathrm{E}^{k} f d m \leq \sigma a \int f d m,
\end{aligned}
$$

for $x$ and $y$ in $\Lambda_{\ell}^{q}, \ell \leq k(j+1)$, using (3.10, 3.11, 3.12),

$$
\begin{aligned}
&\left|\mathrm{E}^{k} f(x)-\mathrm{七}^{k} f(y)\right| \leq d(x, y) \delta_{j} b \int f d m \text { if } \ell \geq k, \\
&\left|\mathrm{七}^{k} f(x)-\mathrm{七}^{k} f(y)\right| \leq d(x, y) \sigma b \int f d m \text { otherwise }
\end{aligned}
$$

for any $x \in P_{2} \cap \Lambda_{\ell}, \ell \leq k(j+1)$, using (3.13] [3.14),

$$
\begin{aligned}
& \left|\mathrm{E}^{k} f(x)\right| \leq \delta_{j} c \int f d m \text { if } \ell \geq k, \\
& \left|\mathrm{E}^{k} f(x)\right| \leq \sigma c \int f d m \text { otherwise. }
\end{aligned}
$$

This concludes the proof since $\sigma<\delta_{j}$.

It is easily seen that all the cones $\mathcal{C}(a, b, c, d, j)$ satisfy the properties of section 2 In order to apply results from section 2, we have to estimate the projective diameter of $\mathrm{E}^{k} \mathcal{C}(a, b, c, d, j)$ into $\mathcal{C}(a, b, c, d, j+1)$ and to find adapted homogeneous form and norm. 
Lemma 3.10. The projective diameter $d_{j}$ of $E^{k} \mathcal{C}(a, b, c, d, j)$ into $\mathcal{C}(a, b, c, d, j+1)$ is bounded above by

$$
2 \log \max \left[\frac{2\left(\alpha^{\prime}+3 / 8 \alpha\right)}{\alpha}, \frac{1+\delta_{j}}{1-\delta_{j}}\right] .
$$

Moreover, the homogeneous form $f \mapsto \int f d m$ is adapted, so is the norm

$$
\|f\|_{e}=\max \left[e\left|\int f d m\right|, m(P)^{-1} \int_{P} f d m \text { for } P \in \mathcal{P},\|f\|_{\infty}\right],
$$

where $e \geq \max \left[c, D_{1} b\right]$.

Proof. The proof is a straightforward computation, (see K,M,S, Li1], Ma1, Ma2] for similar computations).

The following lemma proves that the cones $\mathcal{C}(a, b, c, d, j)$ are far from being empty.

Lemma 3.11. There exists $a_{0}, b_{0}, c_{0}, d_{0}$ such that for all $a \geq a_{0}, b \geq b_{0}$ and $c \geq c_{0}, d \geq d_{0}$ and for all $j \in \mathbb{N}, h \in \mathcal{C}(j)$.

For any $f \in L$, there exists $R(f)>0$ such that $\frac{f}{v}+R(f) h$ belongs to $\mathcal{C}(j)$ for all $j \in \mathbb{N}$. Moreover, $R(f) \leq \mathrm{Ct}\|f\|$.

Proof. Recall that $h=\frac{h_{0}}{v}$ where $h_{0} \in L$ is the fixed point for $\mathrm{E}_{0}$ (see Theorem 1.5). Condition 4 in the definition of the cone is satisfied for $h$ provided $d \geq\left\|h_{0}\right\|$ (recall $m(h)=1$ ). Condition 1 is satisfied for $h$ provided $a \geq 1$. Condition 2 is satisfied for $h$ provided $b \geq L(h)$. Condition 3 is satisfied for $h$ provided $c \geq\|h\|_{\infty}$.

Let $f \in L$, choose

$$
\begin{gathered}
R(f) \geq \frac{\|f\|-d \int \frac{f}{v} d m}{d-\left\|h_{0}\right\|}, \\
R(f) \geq \frac{\mu(P)^{-1} \int_{P} \frac{f}{v} d m-a \int \frac{f}{v} d m}{a-1} \text { for all } P \in \mathcal{P}, \\
R(f) \geq \frac{L\left(\frac{f}{v}\right)-b \int \frac{f}{v} d m}{b} \text { and } R(f) \geq \frac{\left\|\frac{f}{v}\right\|_{\infty}-c \int \frac{f}{v} d m}{c-\|h\|_{\infty}},
\end{gathered}
$$

so $R(f)$ may be chosen such that there exists a constant (independent of $f$ ) such that $R(f) \leq \mathrm{Ct}\|f\|$.

We will denote by $\gamma_{j}=\tanh \frac{d_{j}}{4}$.

Remark 3.12 . If the ratio $v_{j} / v_{j+1}$ goes to 1 when $j$ goes to infinity then, for any $j \in \mathbb{N}$,

$$
\gamma_{j} \leq \frac{v_{j k}}{v_{(j+1) k}}
$$

If the ration $v_{j} / v_{j+1}$ is smaller than some $0<\beta<1$, then $\gamma_{j}<\gamma$ for some $\gamma<1$. 
In what follows, we assume that $a, b, c$ and $d$ are large enough to ensure that $h$ and $\frac{f}{v}+R(f) h$ belong to $\mathcal{C}(j)$ for all $f \in L$. For $j>0$, define $\alpha(j)=v_{1} / v_{k j}$ if the ratio $v_{j} / v_{j+1}$ goes to $1, \alpha(j)=\gamma^{j}$ ( $\gamma$ is given by the above remark) if the ratio $v_{j} / v_{j+1}$ is smaller than some $0<\beta<1$.

Proposition 3.13. Let $f \in \mathcal{C}(1), p=k j+r, r<k$, then

$$
\left\|E^{p} f-h m(f)\right\|_{\infty} \leq \mathrm{Ct} \alpha(j) m(f) .
$$

For $f \in L$, let $f_{v}=\frac{f}{v}$, then

$$
\left\|E^{p} f_{v}-h m\left(f_{v}\right)\right\|_{\infty} \leq \mathrm{Ct} \alpha(j)\|f\| .
$$

Proof. Letting $f$ belong to $\mathcal{C}(1)$, Proposition 3.7 implies that $\mathrm{E}^{j k} f$ and $\mathrm{E}^{j k} h$ belong to $\mathcal{C}(j)$. Applying successively Theorem 2.1, we get

$$
\theta_{\mathcal{C}(j)}\left(\mathrm{E}^{k j} f, \mathrm{E}^{k j} h\right) \leq \gamma_{j} \theta_{\mathcal{C}(j-1)}\left(\mathrm{E}^{k(j-1)} f, \mathrm{E}^{k(j-1)} h\right) \leq \cdots \leq \prod_{i=2}^{j} \gamma_{+i} \cdot \Delta_{1} .
$$

Since the adapted norm \|\|$_{e}$ is equivalent to \|\|$_{\infty}$ and the form $f \mapsto m(f)$ is adapted, we get

$$
\left\|\mathrm{E}^{k j} f-h\right\|_{\infty} \leq \mathrm{Ct} \alpha(j) m(f) .
$$

This inequality leads to the estimate, writing $p=k j+r$ and using that $\sup \mathrm{E}^{r} \mathbf{1} \leq$ $M$, for functions in $\mathcal{C}(1)$. To get the estimate for all functions in $L$, it suffices to apply the previous argument to $\frac{f}{v}+R(f) h$ (use Lemma 3.11).

The decay of correlations follows from Proposition 3.13 by using (1.3) with $\mathrm{L}$ instead of $\mathrm{E}_{0}$ and $m$ instead of $m_{0}$.

Corollary 3.14 (decay of correlations). If $f$ belongs to $L$ and $g \in L^{1}(m)$, then

$$
c_{p}(f, g) \leq \mathrm{Ct} \alpha([p / k])\|f\|\|g\|_{1} .
$$

Proof. It suffices to remember that

$$
c_{p}(f, g)=\int g\left[\mathrm{E}^{p}(f h)-h m(f h)\right] d m
$$

and apply Proposition 3.13 to $f h=\frac{f h_{0}}{v}$ with $f h_{0} \in L$.

We conclude this section with the estimations for functions in $L$ and for the initial transfer operator $\mathrm{E}_{0}$.

Corollary 3.15. For any $f \in L$ and $g$ such that $g v \in L^{1}\left(m_{0}\right)$, for $p \in \mathbb{N}$, and any increasing sequence $\left(v_{\ell}\right)_{\ell \in \mathbb{N}}$ such that $\sum_{\ell} v_{\ell} m_{0}\left(\Lambda_{\ell}\right)<\infty$, for any $\ell \in \mathbb{N}$

$$
\sup _{\Lambda_{\ell}}\left|E_{0}^{p} f-h_{0} m_{0}(f)\right| \leq \mathrm{Ct} v_{\ell} \alpha([p / k])\|f / v\|,
$$

and

$$
c_{p}(f, g) \leq \mathrm{Ct} \alpha([p / k])\|f\|\|g v\|_{1} .
$$

Proof. It suffices to use that $\mathrm{E}_{0} f=v_{\ell} \mathrm{E}(f / v)$ for $x \in \Lambda_{\ell}$.

\section{Further Discussions}

To conclude, we will give some specific rates of mixing and some lower bounds for the speed of convergence in the uniform norm. 
4.1. Exponential decay. If there exist constants $0<e_{1}$ and $0<\theta<1$ such that for all $\ell \in \mathbb{N}, m\left(\Lambda_{\ell}\right) \leq e_{1} \theta^{\ell}$, then it may be taken $v_{\ell}=\Theta^{\ell}$ provided $\Theta^{-1}<\theta$. In this situation, $\delta_{j} \leq \Theta^{-1}<1$. Following Remark 3.6 it is sufficient to work with cones $\mathcal{C}(a, b, c)$. We have: $\mathrm{E}^{k} \mathcal{C}(a, b, c) \subset \mathcal{C}\left(\sigma a, \Theta^{-1} b, \Theta^{-1} c\right)$ and the projective diameter of $\mathrm{E}^{k} \mathcal{C}(a, b, c)$ into $\mathcal{C}(a, b, c)$ is bounded by $2 \log \max \left[\frac{2\left(\alpha^{\prime}+3 / 8 \alpha\right)}{\alpha}, \frac{1+\Theta^{-1}}{1-\Theta^{-1}}\right]$, where $0<\alpha<1<\alpha^{\prime}$ are arbitrary. We obtain exponential decay of correlations for functions in $L$. Moreover the norm \|\|$_{e}^{\prime}=\max \left[\|\|_{e}, L()\right]$ is adapted to the cone $\mathcal{C}(a, b, c)$. This implies that the transfer operator $\mathrm{E}$ on $L$ is quasi-compact. This quasi-compactness result has been proved by L.-S. Young ( $\mathrm{Y1}$ ) by approximating $\mathrm{E}$ with finite rank operators. The cone method gives a constructive bound for the second eigenvalue if it exists.

Some unimodal maps and Henon maps may be conjugating with tower having exponentially small upper floors, that is: $m\left(\Lambda_{l}\right)<e_{1} \theta^{l}$ ([B,Y], [Y1] ).

4.2. Stretched exponential decay. If there exist constants $0<e_{1}, 0<\theta<1$ and $0<\beta<1$ such that for all $\ell \in \mathbb{N}, m\left(\Lambda_{\ell}\right) \leq e_{1} \theta^{\ell^{\beta}}$, then it may be taken $v_{\ell}=\left(\frac{1}{\theta}\right)^{\ell^{\beta^{\prime}}}$ provided $\beta^{\prime}<\beta$. We obtain decay of correlations in $\theta^{n^{\beta^{\prime}}}$ for functions in $L$.

4.3. Polynomial decay. If there exist constants $0<e_{1}, \beta>1$ such that for all $\ell \in \mathbb{N}, m\left(\Lambda_{\ell}\right) \leq e_{1} \ell^{-\beta}$, it may be taken that $v_{\ell}=\frac{\ell^{\beta-1}}{(\ln \ell)^{\gamma}}$ provided $\gamma>1$. We obtain decay of correlations in $\frac{(\ln n)^{\gamma}}{n^{\beta-1}}$ for functions in $L$. L.-S. Young obtained decay of correlations in $1 / n^{\beta-1}$. Our result actually gives

$$
c_{n}(f, g) \leq C \frac{(\ln n)^{\gamma}}{n^{\beta-1}}\|f\|\|g v\|_{1}
$$

while in $\mathrm{Y} 2$ the result is

$$
c_{n}(f, g) \leq C \frac{1}{n^{\beta-1}}\|f\|\|g\|_{\infty} .
$$

The fact that we have a $L^{1}$ norm may be crucial to obtain sharp statistical properties on the system (like asymptotic laws of successive return times, see $\mathrm{C}, \mathrm{G}, \mathrm{S}],[\mathrm{P}]$ ).

4.4. Lower bounds. We use a modification of L.-S. Young's argument ( $\mathrm{Y} 2]$ ). Letting $f=h+c / v_{\ell}$ on $\Lambda_{\ell}$ with $\ell \geq 1, c$ is such that $m(f)=1$. Clearly $f=\frac{f_{0}}{v}$ with $f_{0} \in L$. We have $\mathrm{E}^{n} f=h+c / v_{\ell}$ on $\Lambda_{\ell}$ for $\ell \geq n$. So, for $\ell \geq n, \mathrm{七}^{n} f-h m(f)=c / v_{\ell}$ on $\Lambda_{\ell}$. This implies that the bound given in Proposition 3.13 is optimal if the ratio $v_{j} / v_{j+1}$ goes to 1 .

\section{REFERENCES}

[A,D,U] J. AARONSON, M. DENKER, and M. URBANSKI Ergodic theory for Markov fibered systems and parabolic rational maps. Trans. Amer. Math. Soc. (1993), 337 (2), 495-548. MR 94g:58116

$[\mathrm{Ba}, \mathrm{V}]$ V. BALADI, M. VIANA Strong stochastic stability and rate of mixing for unimodal maps. Ann. Sci. École Norm. Sup. (1996) 29, 4, 483-517. MR 97d:58170

$[\mathrm{Ba}, \mathrm{Y}]$ V.BALADI, L.-S. YOUNG On the spectra of randomly perturbed expanding maps. Comm. Math. Phys. (1993) 156, 2, 355-385. MR 94g:58172

$[\mathrm{B}, \mathrm{Y}]$ M. BENEDICKS and L.-S. YOUNG Decay of correlations for certain Henon maps. (1996) preprint.

[Bi1] G. BIRKHOFF Extensions of Jentzch's theorem. T.A.M.S. (1957), 85, 219-227. MR 19:296a 
[Bi2] G. BIRKHOFF Lattice theory (3rd edition). Amer. Math. Soc. (1967). MR 37:2635

[Buz] J. BUZZI Markov extensions for multi-dimensional dynamical systems. Israel J. Math. 112 (1999), 357-380. MR 2000m:37008

[Ca] A.A. CASTRO Backward inducing and exponential decay of correlations for partially hyperbolic attractors with mostly contracting central direction. $\mathrm{PhD}$ Thesis (1998).

[C] P. COLLET Statistics of closest return for some non uniformly hyperbolic systems. Preprint (1999)

[C,G,S] P. COLLET, A. GALVES, B. SCHMITT Unpredictability of the occurrence time of a long laminar period in a model of temporal intermitency. Ann. Inst. H. Poincaré Phys. Théor. (1992), 57, 3, 319-331. MR 94a:58064

[Ch] N. CHERNOV Statistical properties of piecewise smooth hyperbolic systems in high dimensions. Discrete Contin. Dynam. Systems (1999), 5, 2, 425-448. MR 99k:58134

[D] D. DOLGOPYAT On dynamics of mostly contracting diffeomorphisms. Preprint (1998).

[F,S1] P. FERRERO, B. SCHMITT Ruelle Perron Frobenius theorems and projective metrics. Colloque Math. Soc. J. Bolyai Random Fields. Estergom (Hungary) (1979).

[F,S2] P. FERRERO, B. SCHMITT On the rate of convergence for some limit ratio theorems related to endomorphisms with a non regular invariant density. Preprint (1994).

[G,S] A. GALVES \& B. SCHMITT Inequalities for hitting time in mixing dynamical systems. Random and Computational Dynamics (1997), 5, 4, 337-347. MR 98i:60017

[H] F. HOFBAUER On intrinsic ergodicity of piecewise monotonic transformations with positive entropy. Israel J. Math. (1979), 34, 1, 213-237; (1981), 38, 11, 107-115.

$[\mathrm{K}, \mathrm{N}] \quad$ G. KELLER, T. NOWICKI Spectral theory, zeta functions and the distribution of periodic points for Collet-Eckmann maps. Comm. Math. Phys. (1992), 149, 1, 31-69. MR 93i:58123

[K,M,S] A. KONDAH, V. MAUME, and B. SCHMITT Vitesse de convergence vers l'état d'équlibre pour des dynamiques markoviennes non höldériennes. Ann. Inst. Poincarré Sec. Prob. Stat. (1997) 33 (6) 675-695. MR 99e:28032

[Li1] C. LIVERANI Decay of correlations. Ann. of Math. (1995), 142 (2), 239-301. MR 96e:58090

[Li2] C. LIVERANI Central limit theorem for deterministic systems. Proceedings of the International Congress on Dynamical Systems, Montevideo 95, Research Notes in Mathematics series, Pittman, (1997). MR 98k:28025

[Ma1] V. MAUME-DESCHAMPS Propriétés de mélange pour des systèmes dynamiques markoviens. PhD Thesis, Université de Bourgogne (1998), http://www.u-bourgogne.fr/monge/v.maume/accueil.html

[Ma2] V. MAUME-DESCHAMPS Correlation decay for Markov maps on a countable state space. To appear in Erg. Th Dyn. Syst.

[P] F. PACCAUT Statistics of return times for weighted maps of the interval Preprint (1999).

[Sa] O. SARIG Thermodynamic Formalism for Countable Markov Shifts. 19 (1999), 15651593. MR 2000m:37009

[Sau] B. SAUSSOL Étude statistique de systèmes dynamiques dilatants. PhD. Thesis, Université de Toulon.

[Se] E. SENETA Non-negative matrices and Markov chains. Springer (1981). MR 85i:60058

[V] M. VIANA Stochastic dynamics of deterministic systems. (1997).

[Y1] L.-S. YOUNG Statistical properties of dynamical systems with some hyperbolicity. Ann. of Math. (2) (1998), 147, 3, 585-650. MR 99h:58140

[Y2] L.-S. YOUNG Recurrence times and rates of mixing. Israel J. Math. 110 (1999), 153-188. CMP 2000:11

Département de Mathématiques, Université de Genève, Geneva, Switzerland Current address: Université de Bourgogne, B.P. 47870, 21078 Dijon Cedex, France

E-mail address: vmaume@topolog.u-bourgogne.fr 